\title{
PERFIL DAS MULHERES DIAGNOSTICADAS COM CÂNCER DE MAMA NO MUNICÍPIO DE MARINGÁ-PR
}

Julia Wosch Brochonski

Acadêmica no curso Medicina do Centro Univeristário de Maringá (UNICESUMAR), Brasil.

\section{Sabrina de Almeida Rodrigues}

Acadêmica no curso Medicina do Centro Univeristário de Maringá (UNICESUMAR), Brasil.

\section{Cristiana Aparecida Soares Manzotti}

Mestranda no Programa de Pós-graduação em Promoção da Saúde (PPGPS) do Centro Universitário de Maringá (UNICESUMAR), Brasil.

\section{Marcelo Picinin Bernuci}

Docente no Programa de Pós-graduação em Promoção da Saúde (PPGPS) do Centro Universitário de Maringá (UNICESUMAR); Pesquisador do Instituto Cesumar de Ciência, Tecnologia e Inovação (ICETI), Brasil.
RESUMO: O câncer de mama é uma das neoplasias mais frequentes no mundo e um dos propósitos das instituições de saúde pública é aumentar os índices de diagnóstico precoce. Este estudo teve como objetivo verificar os fatores de risco, estadiamento dos tumores e analisar o tempo de investigação. Estudo descritivo realizado no centro de referência de câncer de mama de Maringá a partir da análise de prontuários de mulheres diagnosticadas com câncer de mama do tipo maligno em 2013 e 2014 . Mais de 30\% das pacientes diagnosticadas estavam fora da faixa etária de rastreamento e o diagnóstico foi tardio em quase $70 \%$. Fatores de risco como obesidade, histórico familiar e uso de terapia de reposição hormonal estavam ausentes na maioria dos casos. O serviço mostrou-se eficaz na realização da biópsia e indicação do tratamento, porém muitos diagnósticos ainda são realizados em estágios avançados da doença sugerindo a necessidade de conscientização da população-alvo à realização dos exames preventivos.

PALAVRAS-CHAVE: Câncer de mama; Saúde da mulher; Diagnóstico precoce.

\section{PROFILE OF FEMALES WITH BREAST CANCER IN MARINGÁ, BRAZIL}

\begin{abstract}
Breast cancer is one of the most common neoplasms worldwide and one of aims of public health institutions is to increase early diagnosis rates. Current descriptive research verifies risk factors, tumor stages and investigation period in a reference center for breast cancer in Maringá PR Brazil through analysis of clinical charts of females diagnosed with malign breast cancer in 2013 and 2014. More than 30\% of patients were beyond the age bracket and late diagnosis occurred in almost $70 \%$ of cases. Risk factors such as obesity, family history and hormonal reposition therapy were absent in most cases. Service was highly efficient in biopsy and indication of treatment. However, most diagnoses were undertaken in the latter stages of the disease. Awareness in the target population for preventive tests is recommended.
\end{abstract}

KEY WORDS: Breast cancer; Women’s health; Early diagnosis. 


\section{INTRODUÇÃO}

As neoplasias malignas apresentam prevalência crescente na população mundial e constituem importante problema de saúde pública, pois estima-se que somente no Brasil sejam diagnosticadas para o biênio 2016-2017 cerca de 600 mil novos casos, sendo que os cânceres de mama correspondem a 28,1\% destes, segundo estimativas do Instituto Nacional de Câncer José Alencar Gomes da Silva (INCA, 2015).

O rastreamento tem como finalidade identificar lesões sugestivas de câncer e encaminhar as pacientes com resultados alterados para exame diagnóstico e tratamento ( $\mathrm{WH} 0,2007$ ), porém, no Brasil o rastreamento do tipo oportunístico ainda prevalece (SILVA; HORTALE, 2012). Os investimentos no rastreamento e diagnóstico precoce, bem como nos reconhecimentos dos fatores predisponentes para o câncer de mama são fundamentais para a redução dos índices de morbidade e mortalidade (OMS, 2014). A implementação do rastreamento organizado do câncer de mama nos municípios, ofertado para as mulheres entre 50 e 69 anos, com realização de mamografia de qualidade a cada dois anos, pode reduzir em até 35\% a mortalidade, desde que seja alcançada cobertura populacional igual ou superior a $70 \%$, pois a cura pode chegar a $100 \%$ quando diagnosticada a doença em estágio precoce (IBCC, 2016).

Entretanto, mais de $60 \%$ dos casos são diagnosticados em estágios avançados, favorecendo a redução da sobrevida, a instituição de tratamento agressivo e perdas na qualidade de vida (SILVA, 2012). Em geral, a sobrevida média de pacientes com câncer de mama é superior em cinco anos nos países desenvolvidos e menor em países em desenvolvimento (COLEMAN et al., 2007), sugerindo que as ações de diagnóstico precoce realizadas de forma organizada são de fato mais eficazes para redução da mortalidade por essa neoplasia.

A análise da presença de fatores de risco tanto modificáveis quanto não modificáveis na população em estudo merece também ser incluído na análise dos fatores associados ao prognóstico da paciente (SILVA; RIUL, 2011). Dentre os fatores não modificáveis destacam-se o histórico familiar, idade avançada, etnia, exposição prolongada a estrógenos endógenos (menarca precoce), menopausa tardia, nenhuma gestação ou primeira após 30 anos e outras comorbidades; e os modificáveis como a obesidade, estilo de vida, sedentarismo, uso de estrógenos exógenos (terapia de reposição hormonal) (TIEZZI, 2013; INCA, 2014; BRASIL, MINISTÉRIO DA SAÚDE 2013).

Diante da relevância da eficácia das ações de rastreamento e diagnóstico precoce para o controle do câncer da mama, preconizados pelo INCA, o presente estudo tem como objetivo caracterizar clinicamente as mulheres diagnosticadas no município de Maringá, verificando se o diagnóstico está sendo precoce ou tardio, o tempo de confirmação diagnóstica e sua influência no estadiamento, além da presença dos fatores de risco associados à doença na população em estudo. Acreditamos que esses resultados são significativos para avaliar a eficácia das estratégias de prevenção e diagnóstico do combate ao câncer de mamas realizadas atualmente no Sistema Único de Saúde, principalmente naquelas regiões onde as mulheres estão sendo diagnosticadas em períodos cada vez mais tardios.

\section{METODOLOGIA}

Trata-se de um estudo descritivo do tipo retrospectivo, cujo objetivo foi estudar e descrever as características epidemiológicas, estadiamento e fatores de risco associados ao câncer de mama nas mulheres com diagnóstico CID 10 (Classificação Internacional de Doenças) registrado no programa Gestor do município de Maringá-PR, no período de janeiro de 2013 a dezembro de 2014. O estudo foi aprovado pelo Comitê de Ética em pesquisa do Centro Universitário de Maringá - UniCesumar (protocolo número: 1.092.279).

Os dados foram obtidos por meio do prontuário eletrônico nos arquivos digitais da Clínica da Mulher. Para caracterizar epidemiologicamente a população, complementamos os dados ausentes do prontuário eletrônico por meio de aplicação de questionário semiestruturado, após assinatura do termo de consentimento livre e esclarecido (TCLE). Das 77 pacientes incluídas na análise dos prontuários foram excluídas do estudo com questionários as pacientes residentes nos municípios diferentes de Maringá ( $\mathrm{n}=$ 24); não localizadas ( $n=16)$; mulheres que evoluíram para óbito $(n=1)$; mudaram-se $(n=4)$; ou que se recusaram a participar do estudo $(n=4)$.

Para avaliar a rapidez no diagnóstico e a influência do tempo de investigação no estadiamento, 
verificamos as datas do exame de imagem alterado e da biópsia confirmatória. Esse período foi relacionado com o estágio do tumor e avaliado em duas situações:

- $1^{\text {a }}$ situação: realizamos a comparação entre dois grupos distintos: residentes no município de Maringá e pacientes residentes em municípios vizinhos que foram referenciadas à Clínica da Mulher para investigação. Para verificar se existiu diferença da média de tempo entre o exame de imagem e a biópsia nos dois grupos, foi realizada investigação aplicando-se o teste não paramétrico de Wilcoxon, indicado em situações em que as suposições realizadas nos testes paramétricos não são verificadas.

- $2^{\mathrm{a}}$ situação: verificar se o período de investigação interferiu no estadiamento do tumor, ou seja, se atrasos na investigação foram relevantes para a sobrevida da paciente. Nesta etapa, o tempo foi dividido em quatro categorias (abaixo de 30 dias; entre 30 e 90 dias; entre 90 e 120 dias e acima de 120 dias de investigação) e utilizou-se o teste exato de Fisher. O nível de significância fixado foi de 5\%.

Para verificar a presença dos fatores de risco na população em estudo, os dados socioculturais, econômicos, histórico médico e familiar das pacientes foram extraídos do questionário e entrevista.

\section{RESULTADOS}

\subsection{CARACTERIZAÇÃO DA POPULAÇÃO DIAGNOSTICADA}

Entre as participantes entrevistadas a maioria é branca (64,28\%), estudou até o primeiro grau (50\%), é casada $(42,85 \%)$ e possui renda familiar entre dois e três salários mínimos (64,28\%). Praticam atividade física 46,42\%. Quanto às patologias associadas, 35,71\% referem tratamento para diabetes mellitus (DM), 35,71\% para hipertensão arterial sistêmica, $25 \%$ são obesas e $28,57 \%$ apresentam patologia psicológica em tratamento. Dessas mulheres, 32,13\% estavam fora da faixa etária de rastreamento por meio do exame mamográfico, preconizado pelo Ministério da Saúde, atualmente recomendado de 50 a 69 anos (dados não mostrados).
3.2 FATORES DE RISCO PARA CA DA MAMA NA POPULAÇÃO ENTREVISTADA

Considerando o histórico ginecológico, a maioria das pacientes nunca realizou terapia de reposição hormonal $(96,42 \%)$, não apresentou menarca precoce (96,42\%) ou menopausa tardia (78,57\%) e gestaram duas ou mais vezes (75\%). Com relação aos familiares, apenas $25 \%$ possuem casos de CA de mama em parentes de primeiro grau e $92,30 \%$ das filhas e $83,34 \%$ das irmãs com idade acima de 40 anos realizam exames de rotina para diagnóstico de CA de mama (Tabela 1).

Tabela 1. Histórico ginecológico das mulheres entrevistadas

(Continua)

\begin{tabular}{ccc}
\hline Fator & N & $\%$ \\
\hline Terapia Hormonal & \\
\hline Sim & 1 \\
Não & 27 \\
\hline
\end{tabular}

\begin{tabular}{lcc}
\hline Idade $^{\mathbf{a}}$. MMG & & \\
\hline Até 35 anos & 6 & 21,4 \\
36-49 anos & 11 & 39,3 \\
50-69 anos & 6 & 21,4 \\
$>69$ anos & 3 & 10,7 \\
Indeterminado & 2 & 7,1 \\
\hline Menarca precoce & & \\
\hline Não & 27 & 96,4 \\
Sim & 1 & 3,6 \\
\hline Menopausa tardia & & \\
\hline Sim & 1 & 3,6 \\
Não & 22 & 78,6 \\
Indet./cirúrgica & 5 & 17,9 \\
\hline Gestações & \multicolumn{2}{c}{} \\
\hline Nenhuma & 2 & 7,1 \\
Uma & 5 & 17,9 \\
Duas ou mais & 21 & 75,0 \\
\hline Total & $\mathbf{2 8}$ & $\mathbf{1 0 0 , 0 0}$ \\
\hline Fator & $\mathbf{N}$ & $\%$ \\
\hline
\end{tabular}

Histórico Familiar +

$\begin{array}{lll}\text { Sim } & 7 & 25,0\end{array}$

$\begin{array}{lll}\text { Não } & 21 & 75,0\end{array}$

\begin{tabular}{lcc}
\hline Investigação das filhas & & \\
\hline Sim & 12 & 42,9 \\
Não & 1 & 3,6 \\
\hline
\end{tabular}


(Conclusão)

\begin{tabular}{lcc}
\hline Fator & $\mathbf{N}$ & $\%$ \\
\hline Não se enquadra & 15 & 53,6 \\
\hline Investigação das irmãs & & \\
\hline Sim & 20 & 71,4 \\
Não & 2 & 7,1 \\
Não se enquadra & 4 & 14,3 \\
Desconhece & 2 & 7,1 \\
\hline Total & $\mathbf{2 8}$ & $\mathbf{1 0 0 , 0}$ \\
\hline
\end{tabular}

Fonte: Dados da pesquisa

$\mathrm{Na}$ Tabela 2 estão relacionados os dados sobre o diagnóstico, tratamento e acompanhamento. Somente 28,57\% das pacientes foram diagnosticadas precocemente (Estágio I), 32,14\% necessitaram de cirurgia radical (mastectomia), $71,42 \%$ realizaram quimioterapia/ radioterapia e $39,28 \%$ fizeram ou fazem hormonioterapia. Acompanham periodicamente com mastologista ou oncologista $78,57 \%$, e $\mathbf{1 7 , 8 5 \%}$ estavam em tratamento com radioterapia, com doença recidivada ou metástases na ocasião da entrevista.

Tabela 2. Histórico ginecológico das mulheres entrevistadas

(Continua)

\begin{tabular}{lcc}
\hline Fatores & Freq. & $\%$ \\
\hline Estadiamento & & \\
\hline Estágio I & 8 & 28,6 \\
Estágio II & 10 & 35,7 \\
Estágio III e IV & 9 & 32,1 \\
Indeterminado & 1 & 3,57 \\
\hline Tratamento cirúrgico & & \\
\hline Mastectomia & 9 & 32,1 \\
Cirurgia conservadora & 13 & 46,4 \\
Não cirúrgico/indet. & 6 & 21,4 \\
\hline Tratamento complementar & & \\
\hline Radioterapia & 2 & 7,14 \\
Quimioterapia & 5 & 17,9 \\
Quimioterapia e radioterapia & 20 & 71,4 \\
$\quad$ Não realizaram & 1 & 3,57 \\
\hline Realização de hormonioterapia & \multicolumn{2}{|c}{} \\
\hline Sim & 11 & 39,3 \\
Não & 14 & 50 \\
Indeterminado & 3 & 10,7 \\
\hline Estado de saúde atual & \multicolumn{2}{|c}{} \\
\hline Seguimento ambulatorial & & \\
\hline
\end{tabular}

Tabela 3. Distribuição de frequências entre o tempo exame de imagem alterado e a realização de biópsia de acordo com o estadiamento do tumor

\begin{tabular}{lccccc}
\hline \multirow{2}{*}{ Tempo } & \multicolumn{5}{l}{ Estadiamento } \\
\cline { 2 - 6 } & I & II & III & IV & Indet. \\
\hline$\leq 30$ dias & $3(19 \%)$ & $6(18 \%)$ & $3(25 \%)$ & $1(33 \%)$ & $3(25 \%)$ \\
30 a 90 dias & $8(50 \%)$ & $16(47 \%)$ & $5(42 \%)$ & $1(33 \%)$ & $4(33 \%)$ \\
90 a 120 dias & $2(13 \%)$ & $3(9 \%)$ & $2(17 \%)$ & $0(0 \%)$ & $1(8 \%)$ \\
$>120$ dias & $0(0 \%)$ & $5(15 \%)$ & $0(0 \%)$ & $1(33 \%)$ & $3(25 \%)$ \\
Indeterminado & $3(19 \%)$ & $4(12 \%)$ & $2(17 \%)$ & $0(0 \%)$ & $1(8 \%)$ \\
\hline \multirow{2}{*}{ Total } & 16 & 34 & 12 & 3 & 12 \\
& $(100 \%)$ & $(100 \%)$ & $(100 \%)$ & $(100 \%)$ & $(100 \%)$ \\
\hline
\end{tabular}

Fonte: Dados da pesquisa

Figura 1 mostra que a mediana dos tempos entre o exame de imagem e a realização da biópsia para os pacientes de Maringá e dos demais municípios foi semelhante (65 e 57 dias, respectivamente). Entretanto, observa-se maior variabilidade desta mesma variável no município de Maringá, apresentando inclusive alguns 
valores discrepantes, o que influencia a média, que é relativamente maior que a média dos outros municípios (94,1 e 56,4 dias, respectivamente). Não houve evidências suficientes para afirmar que há diferenças significativas entre os tempos dos pacientes de Maringá e dos outros municípios.

Em relação ao tempo entre a biópsia e a realização da cirurgia, nota-se que tanto a média quanto a mediana dos tempos para os indivíduos residentes em Maringá e nos outros municípios são próximas (médias de 103,4 e 105,2 dias e medianas de 90 e 98 dias, respectivamente).De acordo com o teste de Wilcoxon, não há indícios de que a distribuição do tempo entre a biópsia e a realização da cirurgia difere significativamente para os pacientes de Maringá e dos outros municípios.

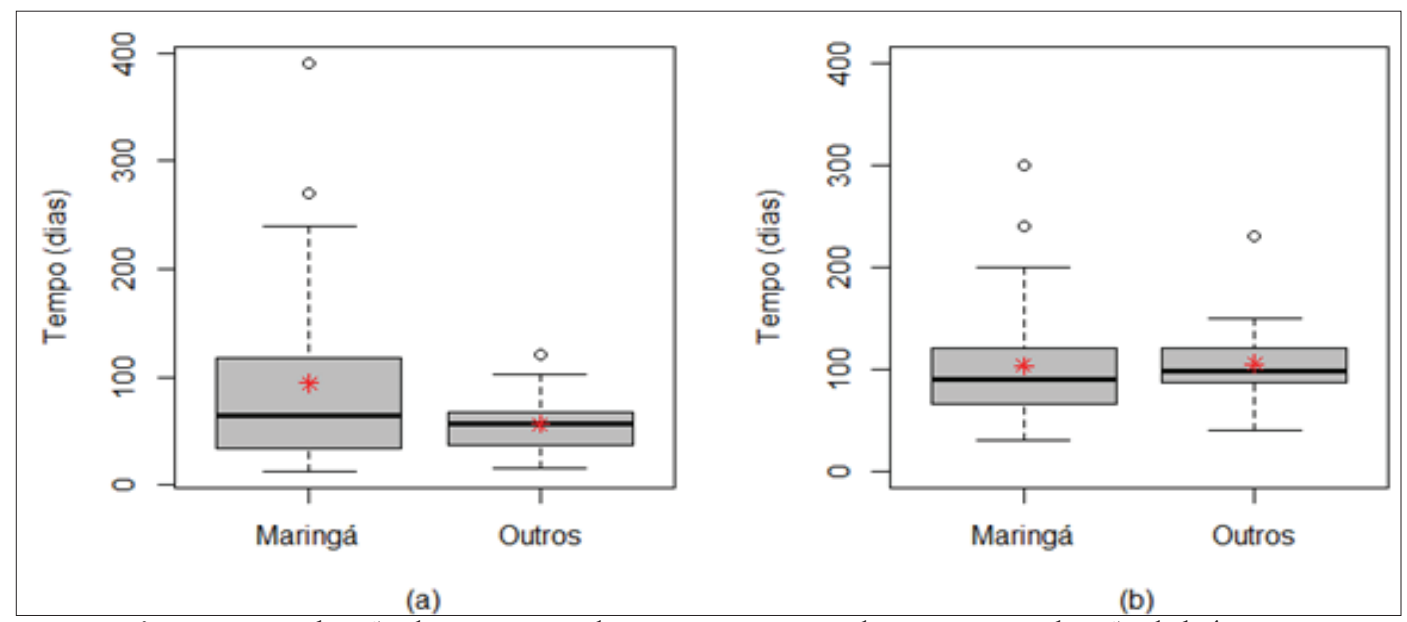

Figura 1. Distribuição do tempo, em dias, entre (a) exame de imagem e realização da biópsia e (b) biópsia e realização da cirurgia, de acordo com o município do paciente.

\subsection{AVALIAÇÃO DA INFLUÊNCIA ENTRE O TEMPO DE INVESTIGAÇÃO E O ESTADIAMENTO DO TUMOR}

Pela Tabela 4, observa-se que não há evidências suficientes que apontam a associação entre o tempo até a realização da biópsia ser menor ou maior que 90 dias e o estágio do tumor ser igual ou superior ao primeiro.

Tabela 4. Distribuição de frequências e resultado do teste exato de Fisher para a associação entre o tempo exame de imagem alterado e a realização de biópsia de acordo com o estadiamento do tumor

\begin{tabular}{lcccc} 
& Estadiamento & & Teste exato de Fisher & Valor $\boldsymbol{p}$ \\
\cline { 2 - 4 } Tempo & I & $\geq$ II & 0,7123 \\
\hline 90 dias & 11 & 32 & 11 & \\
$>90$ dias & 2 & 11 & \\
\hline
\end{tabular}

Fonte: Dados da pesquisa 


\section{DISCUSSÃO}

No Brasil, a taxa de mortalidade por câncer de mama aumentou nos últimos anos, principalmente pelo diagnóstico tardio, pois a patologia é considerada curável se diagnosticada e tratada precocemente. A populaçãoalvo do estudo não foge à regra, com diagnóstico tardio e necessidade de instituição de tratamento agressivo. A detecção precoce do câncer de mama em sua fase inicial é a forma mais eficaz de controle da doença $(\mathrm{KOCH}$, 2010). Sendo assim, é necessário que o equipamento mamográfico seja adequado, assim como uma equipe médica e técnica preparada para a realização e execução da mesma (GOTO, 2013).

Nosso estudo mostrou que a investigação na clínica da mulher foi realizada em um curto período quando comparado aos trabalhos semelhantes realizados em outros serviços, e que o tempo de investigação não afetou o estadiamento. Porém, como na maioria dos casos, o diagnóstico foi realizado tardiamente, esses dados sugerem que há a necessidade de aprimorar as etapas de rastreamento em nível primário, ou seja, aumentar o número de mulheres na faixa etária recomendada para a investigação que é submetida às consultas e mamografias (MS, 2014).

A grande relevância em realizar o diagnóstico precoce encontra-se na possibilidade de cura, pois quando o tratamento é estabelecido em estágio inicial (lesão menor que $2 \mathrm{~cm}$ de diâmetro) determina $o$ prognóstico favorável e a cura pode chegar a $100 \%$ (SILVA, 2012; HORTALE, 2012; BRAY et al., 2004). Estima-se que a taxa de sobrevida global em cinco anos é $98 \%$ para mulheres diagnosticadas em estágio I, 90\% a $82 \%$ estágio II, $60 \%$ estágio III e de $30 \%$ para estágio IV (TIEZZI, 2013). Considerando a importância do diagnóstico precoce, o período médio entre o exame de imagem alterado e a consulta com o mastologista foi 38,77 dias acima nas mulheres residentes em Maringá quando comparado ao das pacientes residentes em outros municípios encaminhadas para investigação. Sabendo que a partir da consulta com o especialista na Clínica da Mulher as biópsias ocorriam, em média, dez dias, e que a instituição do tratamento cirúrgico foi semelhante nos dois grupos, supõe-se que o encaminhamento das mulheres com mamografia ou ultrassom alterado da UBS para o especialista ocorreu de modo efetivo nos pequenos municípios, quando comparado ao mesmo procedimento no município de Maringá.

Embora a diferença entre o tempo de encaminhamento para o especialista não se mostrou estatisticamente significativa, justificado talvez pelo baixo número de pacientes incluídas no estudo, dados mostram a tendência de aumento, o que aponta uma lacuna entre a realização dos exames e a captação das mulheres sob suspeita de câncer da mama, quando comparamos Maringá com outros municípios. Esse período prolongase possivelmente pelas dificuldades institucionais $\mathrm{e}$ falta de ferramentas a fim de localizar as mulheres com exames alterados, visto que existe dissociação entre os prontuários do sistema gestor e os laudos radiológicos e biópsias.

Possivelmente a falta de informação, medo e temor das consequências do tratamento são fatores que interferem na procura das pacientes pelas unidades de saúde, contudo somente $25 \%$ dos casos de atraso no diagnóstico podem ser atribuídos à própria mulher (CAPLAN et al., 1996), portanto, a responsabilidade do diagnóstico tardio cabe aos serviços de saúde, seja na orientação da população para a procura pelos recursos preventivos e diagnósticos, quanto na facilitação ao acesso da população e busca efetiva principalmente nas áreas com altas taxas de ocorrência da doença (BRASIL, MINISTÉRIO DA SAÚDE, 2011; FERREIRA; LF 2011). Uma possível causa para o elevado índice de diagnóstico tardio encontrado em nosso trabalho está relacionada às dificuldades no acesso e cobertura inapropriada do rastreamento mamográfico. A realização adequada na classificação das categorias BI-RADS e de biópsias percutâneas contribui para a detecção precoce $\mathrm{e}$ diminuição da mortalidade do câncer de mama (BADAN, 2013).

Foi possível observar o menor nível de escolaridade e que os fatores socioeconômicos são influentes no aumento da incidência de casos de mortes por câncer mamário, supondo que o contexto social é determinante para várias doenças, como no câncer (MELO, 2013). A promoção e a prevenção em saúde são mais efetivas na população que possua maior informação 
e compreensão sobre os riscos e os métodos de prevenção acerca das doenças. O diagnóstico precoce ainda é uma das formas mais efetivas de prevenção da neoplasia mamária e suas consequências, e fica evidente destacar que os fatores socioeconômicos estão diretamente associados à realização de exames preventivos e a busca pela própria prevenção como o autoexame de mama e mamografias (MELO, 2013). Sendo assim, torna-se necessário maior engajamento da equipe de saúde e gestores responsáveis por essa população, comprometendo-se com o rastreamento correto e precoce, assim como a conduta adequada para cada situação (RONCHI, 2014).

Para tanto, acreditamos que modificações na estrutura do serviço, como inclusão do número do prontuário do sistema gestor do município ao laudo das mamografias, podem facilitar a localização e o contato com as clientes, facilitando o rastreamento e identificação dos exames na UBS, contando com as unidades de PSF já estruturadas para a busca ativa desta população. Além disso, estabelecer parcerias entre as instituições de pesquisa e de saúde pública municipal, estadual e federal, a fim de desenvolver estratégias para abordagem da população-alvo das campanhas para câncer da mama, é outra possibilidade para aumentar o diagnóstico precoce. Esta é uma tarefa complexa, pois existem barreiras institucionais e poucas ferramentas aplicáveis para realizar busca ativa das mulheres em questão.

\section{CONCLUSÃO}

O serviço analisado no estudo mostrou-se eficaz na realização da biópsia e indicação do tratamento, de acordo com o que preconiza o Ministério da Saúde. Entretanto, as altas taxas de diagnóstico em estágios avançados sugerem a necessidade de que os gestores busquem medidas que favoreçam a conscientização da população-alvo para buscar atendimento precoce e garantir que o mesmo aconteça com os encaminhamentos, aumentando o diagnóstico precoce da neoplasia mamária e reduzindo sua morbidade e mortalidade.

\section{REFERÊNCIAS}

BADAN, G. Complete internal audit of a mammography service in a reference institution for breast imaging. Radiol Bras, São Paulo, v. 47, n. 2, p. 74-78, apr. 2014. Disponível em: $\quad<$ http://www.scielo.br/scielo.php?script $=$ sci arttext\&pid $=$ S0100-39842014000200007\&lng =en\&nr $\mathrm{m}=$ iso $>$. Acesso em: 14 mar. 2014.

BRAY, F.; McCARRON, P.; PARKIN, D. The changing global patterns of female breast cancer incidence and mortality. Breast Cancer Res.v.6, n.6, p. 229-39. 2004.

BRASIL. Ministério da Saúde. Secretaria de Atenção à Saúde. Departamento de Atenção Básica. Controle dos cânceres do colo do útero e da mama. 2. ed. Brasília: Ministério da Saúde, 2013. Disponível em: <http:// bvsms.saude. gov.br/bvs/publicacoes/controle_canceres_ colo_utero_2013.pdf>. Acesso em: 14 mar. 2016.

BRASIL. Ministério da Saúde. Mais saúde: direito de todos 2008-2011. 5. ed. Brasília: Ministério da Saúde; 2010 164p. Disponível em: <http:// bvsms.saude.gov.br/ bvs/publicacoes/mais_saude_direito_todos_3ed.pdf $>$. Acesso em: 14 mar. 2016

CAPLAN L.S et al. Reasons for delay in breast cancer diagnosis. Prev Med., v.25, n. 2, p. 218-224, mar./apr. 1996. Disponível em: <http://www.sciencedirect.com/ science/article/pii/S0091743596900493?via\%3Dihub>.

COLEMAN, M.P et al. Cancer survival in five continents: a wordwide population-based study (CONCORD). Lancet Oncol, v. 9, n. 8, p. 730-56, 2008.

FERREIRA, L.F.F.; FERNANDES, S.S.; PETEL, L.A. The natural history of breast cancer in the young patient: literature revision. Femina, v. 39, n. 11, nov. p. 527-531， 2011. Disponível em: <http://files.bvs.br/ upload/S/0100-7254/2011/v39n11/ a2970.pdf >. Acesso em: 14 mar. 2014.

GOTO, R.; PIRES, S; MEDEIROS, R. Identificação de parâmetros de qualidade de impressão para a garantia da detecção de estruturas presentes na mamografia digital. Radiol Bras, São Paulo, v. 46, n. 3, p. 156162, June 2013. Disponível em: <http://www.scielo. 
br $/$ scielo.php?script $=$ sci_arttext $\&$ pid $=S 0100$ $39842013000300156 \& \operatorname{lng}=$ en\&nrm $=$ iso $>$. Acesso em: 14 mar. 2014.

INCA. Instituto Nacional do Câncer. Tipos de câncer. 2015. Disponível em: <http://www2.inc.gob.br/wps/ wcm/connet/tiposdecancer $>$. Acesso em: 14 mar. 2014.

INCA. Instituto Nacional do Câncer. Rastreamento organizado do câncer da mama. 2014. Disponível em: $<$ http://www1.inca.gov.br > . Acesso em: Acesso em: 14 mar. 2014.

INSTITUTO BRASILEIRO DE CONTROLE DO CÂNCER. Mastologia: câncer de mama. 2016 Disponível em :http:// www.ibcc.org.br/duvidas-frequentes/especialidadesmedicas/mastologia.asp.

INSTITUTO NACIONAL DE CÂNCER JOSÉ ALENCAR GOMES DA SILVA. Diretrizes para a detecção precoce do câncer de mama no Brasil. Rio de Janeiro, 2015. Disponível em: < http://www1.inca.gov.br/inca/Arquivos/ livro_deteccao_precoce_final.pdf $>$. Acesso em: 14 mar. 2014.

KOCH, H.; CASTRO, M.V.K. Qualidade da interpretação do diagnóstico mamográfico. Radiol Bras, São Paulo, v. 43, n. 2, p. 97-101, Apr. 2010. Disponível em:<http://www. scielo.br/scielo.php?script $=$ sci_arttext\&pid $=S 0100$ $39842010000200009 \& \operatorname{lng}=$ en\&nrm $=$ iso $>$. Acesso em: 14 mar. 2014.

MELO, W.; SOUZA, L.; ZURITA, R.; CARVALHO, M. Fatores associados na mortalidade por câncer de mama no noroeste paranaense. Revista Eletrônica Gestão e Saúde, v. 1, n. 1, p. 1809-1816, mar. 2013. Disponível em: $\quad<$ http://www.gestaoesaude.unb.br/index.php/ gestaoesaude/article/view/437>. Acesso em: 14 mar. 2014

MINISTÉRIO DA SAÚDE. Caderno de Diretrizes, Objetivos, Metas e Indicadores: 2013-2015. 2. ed. Brasília: Ministério da Saúde, 2014. Disponível em: <www.saude.gov.br/bvs>. Acesso em: 14 mar. 2014.

OMS. Organização Mundial da Saúde. World Health Statistics 2014. Disponível em: < http://www.int/gho/ publications/world_health_statistics/2014/en/>. Acesso em: 14 mar. 2014.

RONCHI, S. Prevalência de alterações mamárias em mulheres atendidas em um município do estado do Paraná. Rev. Gaúcha Enferm., Porto Alegre, v. 35, n. 2, p. 113-120, june 2014. Disponível em: <http://www. scielo.br/scielo.php?script $=$ sci_arttext\&pid $=$ S1983$14472014000200113 \& \operatorname{lng}=$ en\&nrm $=$ iso $>$. Acesso em: 14 mar. 2014.

SILVA, P.A.; RIUL, S. Câncer de mama: fatores de risco e detecção precoce. Rev. Bras. Enferm, Brasília, v. 64, n. 6, p. 1016-1021, nov./dez. 2011. Disponível em: < http:// www.scielo.br/pdf/reben/v64n6/v64n6a05.pdf $>$. Acesso em: 14 mar. 2014.

SILVA. R. C. F.; HORTALE, V. A. Rastreamento do câncer de mama no Brasil: quem, como e por quê? Rev Bras Cancerol., n. 58, v. 1, p. 67-71, 2012. Disponível em: $<$ http://www.inca.gov.br/rbc/n_58/v01/pdf/10b_artigo_ opiniao_rastreamento_cancer_mama_brasil_quem_ como_por_que.pdf>. Acesso em: 14 mar. 2014.

TIEZZI, D. G. Rastreamento do câncer de mama no Brasil: ainda há tempo para refletirmos. Rev. Bras. Ginecol. Obstet., Rio de Janeiro, v. 35, n. 9, p. 385387, sept. 2013 . Disponível em: <http://www. scielo.br/scielo.php?script $=$ sci_arttext\&pid $=S 0100$ $72032013000900001 \& \operatorname{lng}=\mathrm{en} \& \mathrm{nrm}=\mathrm{iso}>$. Acesso em: 14 mar. 2014.

WORLD HEALTH ORGANIZATION. Cancer control: knowledge into action: WHO guide for effective programmes. Prevention Geneva, 2007. Disponível em: $<$ http://www.who.int/cancer/modules/Prevention\%20 Module.pdf $>$. Acesso em: 14 mar. 2014

Recebido em: 10 de outubro de 2016 Aceito em: 30 de março de 2017 